Atmos. Chem. Phys., 18, 3419-3431, 2018

https://doi.org/10.5194/acp-18-3419-2018

(C) Author(s) 2018. This work is distributed under

the Creative Commons Attribution 4.0 License.

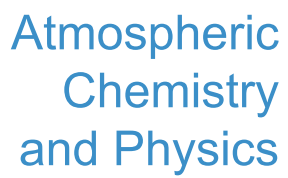

(c) (P)

\title{
The vapor pressure over nano-crystalline ice
}

\author{
Mario Nachbar ${ }^{1,2}$, Denis Duft ${ }^{2}$, and Thomas Leisner ${ }^{1,2}$ \\ ${ }^{1}$ Institute of Environmental Physics, University of Heidelberg, Im Neuenheimer Feld 229, 69120 Heidelberg, Germany \\ ${ }^{2}$ Institute of Meteorology and Climate Research, Karlsruhe Institute of Technology - KIT, Hermann-von-Helmholtz-Platz 1, \\ 76344 Eggenstein-Leopoldshafen, Germany
}

Correspondence: Mario Nachbar (mario.nachbar@kit.edu)

Received: 27 November 2017 - Discussion started: 5 December 2017

Revised: 1 February 2018 - Accepted: 2 February 2018 - Published: 8 March 2018

\begin{abstract}
The crystallization of amorphous solid water (ASW) is known to form nano-crystalline ice. The influence of the nanoscale crystallite size on physical properties like the vapor pressure is relevant for processes in which the crystallization of amorphous ices occurs, e.g., in interstellar ices or cold ice cloud formation in planetary atmospheres, but up to now is not well understood. Here, we present laboratory measurements on the saturation vapor pressure over ice crystallized from ASW between 135 and $190 \mathrm{~K}$. Below $160 \mathrm{~K}$, where the crystallization of ASW is known to form nano-crystalline ice, we obtain a saturation vapor pressure that is 100 to $200 \%$ higher compared to stable hexagonal ice. This elevated vapor pressure is in striking contrast to the vapor pressure of stacking disordered ice which is expected to be the prevailing ice polymorph at these temperatures with a vapor pressure at most $18 \%$ higher than that of hexagonal ice. This apparent discrepancy can be reconciled by assuming that nanoscale crystallites form in the crystallization process of ASW. The high curvature of the nano-crystallites results in a vapor pressure increase that can be described by the Kelvin equation. Our measurements are consistent with the assumption that ASW is the first solid form of ice deposited from the vapor phase at temperatures up to $160 \mathrm{~K}$. Nano-crystalline ice with a mean diameter between 7 and $19 \mathrm{~nm}$ forms thereafter by crystallization within the ASW matrix. The estimated crystal sizes are in agreement with reported crystal size measurements and remain stable for hours below $160 \mathrm{~K}$. Thus, this ice polymorph may be regarded as an independent phase for many atmospheric processes below $160 \mathrm{~K}$ and we parameterize its vapor pressure using a constant Gibbs free energy difference of $(982 \pm 182) \mathrm{J} \mathrm{mol}^{-1}$ relative to hexagonal ice.
\end{abstract}

\section{Introduction}

It is well known that the crystallization process of amorphous solid water (ASW) below about $160 \mathrm{~K}$ forms nano-crystalline ice with crystallite diameters between 5 and $40 \mathrm{~nm}$. Using electron diffraction, Jenniskens and Blake (1996) observed crystal diameters of 10 to $15 \mathrm{~nm}$ between 150 and $160 \mathrm{~K}$ and Kumai (1968) reported diameters of 5 to $30 \mathrm{~nm}$ at 113 to $143 \mathrm{~K}$. Dowell and Rinfret (1960) used X-ray diffraction and observed grain sizes of about $40 \mathrm{~nm}$. Crystallization of the high-pressure ices II, IV, V and IX has been shown to produce nano-crystalline ice as well (Arnold et al., 1968; Kuhs et al., 1987). This nano-granular structure may have significant effects on the properties of the ice polymorph. For example, Johari and Andersson (2015) attributed a reduction in the measured thermal conductivity of ice crystallized from ASW to enhanced phonon scattering at stacking faults and grain boundaries of the crystallites. Furthermore, the nano-crystallites might impact the vapor pressure over the ice phase, but to the best of our knowledge, this effect has not been quantified yet.

Below $160 \mathrm{~K}$, nano-crystalline ice is stable for several hours (Hansen et al., 2008) and thus its vapor pressure is of relevance for atmospheric processes occurring in these conditions, e.g., cloud formation processes in the terrestrial mesosphere or on other planets like Mars. At temperatures below $160 \mathrm{~K}$, however, only a limited number of desorption rate measurements of ice crystallized from ASW using quadrupole mass spectrometers and quartz crystal microbalances is available that may be used to calculate the saturation vapor pressure over the ice phase (Brown et al., 1996; Bryson et al., 1974; Fraser et al., 2001; La Spisa et al., 2001; Sack and Baragiola, 1993; Smith et al., 2011; Speedy et al., 1996). 
Measuring water vapor desorption rates at such low temperatures is a challenging task and these measurements reveal large discrepancies among each other. This situation points to the need for high-quality saturation vapor pressure measurements of nano-crystalline ice crystallized from ASW.

In this work, we report the vapor pressure of ice samples deposited from the gas phase below $160 \mathrm{~K}$ in a temperature range between 135 and $190 \mathrm{~K}$ using two independent and complementary experimental setups. One setup is based on a technique for measuring absolute saturation vapor pressures using the growth of trapped nanoparticles in isothermal conditions as a sensitive probe at temperatures between 135 and $160 \mathrm{~K}$. This setup is briefly described in Sect. 2.1. In order to extend the range to temperatures around $190 \mathrm{~K}$, for which the vapor pressure of crystalline ice is established within a few percent, we also report results from an independent more conventional setup. It allows for the measurement of the relative vapor pressure of water ice samples with respect to hexagonal ice $I_{\mathrm{h}}$ using temperature ramping in the range between 166 and $190 \mathrm{~K}$ and is detailed in Sect. 2.2. In Sect. 3, we present our results from both setups. In Sect. 4, we discuss our results and compare them with the literature.

\section{Experimental design}

\subsection{Isothermal vapor pressure measurements using MICE-TRAPS $(T=135-160 \mathrm{~K})$}

The molecular flow ice cell within the trapped reactive atmospheric particle spectrometer (MICE-TRAPS; Duft et al., 2015; Meinen et al., 2010) has been used previously to investigate the adsorption and nucleation of $\mathrm{CO}_{2}$ on levitated small metal-oxide nanoparticles at low temperatures (Nachbar et al., 2016). In this work, we expose the nanoparticles to a flow of water molecules originated from temperature-controlled ice-covered sample surfaces. We utilize the growth of the nanoparticles as a sensitive probe for the sublimation rate of water molecules and thus the saturation vapor pressure over the ice-covered sample surfaces. In the following, we briefly describe the experimental setup and introduce the experimental procedure applied in this study.

We generate single-charge silica $\left(\mathrm{SiO}_{2}, \rho=2.3 \mathrm{~kg} \mathrm{~m}^{-3}\right)$ and iron oxide $\left(\mathrm{Fe}_{2} \mathrm{O}_{3}, \rho=5.2 \mathrm{~kg} \mathrm{~m}^{-3}\right)$ particles with a radius smaller than $4 \mathrm{~nm}$ in a nonthermal, low-pressure microwave plasma particle source (Nachbar et al., 2018). The nanoparticles are transferred into TRAPS, a low-pressure vacuum apparatus in which they are size selected and stored in MICE, which is a combination of a linear ion trap and a supersaturation cell operating in the molecular flow regime (Duft et al., 2015). An illustration of the radial cross section of MICE is shown in Fig. 1. In MICE, the charged nanoparticles are trapped in the center between the four quadrupole ion trap electrodes (1) where the nanoparticles are exposed to an adjustable supersaturation of $\mathrm{H}_{2} \mathrm{O}$

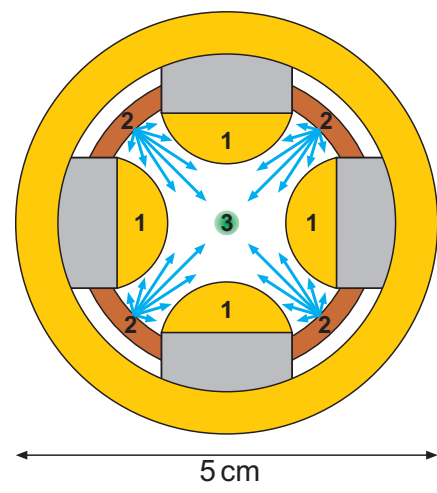

1) Ion trap electrodes $\left(T_{e}\right)$

2) Ice sample surface $\left(T_{s}\right)$

3) Particle cloud $\left(T_{p}\right)$

Figure 1. Radial cross section of MICE.

molecules. This is achieved by temperature-controlled sublimation from two sets of ice-covered gold surfaces, which are held at two distinct temperatures and which completely surround the levitated particles. These surfaces are the ion trap electrodes held at temperature $T_{\mathrm{e}}$ and the ice-covered sample surfaces between the electrodes, which can be heated to an offset temperature $T_{\mathrm{s}}$. The temperature of the nanoparticles equilibrates by collisions with a helium background gas and can be calculated as described in Duft et al. (2015). Before the start of each measurement series, a layer of ice several micrometers thick is deposited from the gas phase at variable sample surface temperatures between 95 and $160 \mathrm{~K}$ with a deposition rate between 1 and $10 \mathrm{~nm} \mathrm{~s}^{-1}$. Deposition is performed while evacuating the TRAPS chamber and water vapor is provided from a water reservoir containing Nanopure $^{\text {TM }}$ water. After deposition, the ice-covered electrodes and additional surfaces are set to the desired temperature such that saturation in excess of $S=1000$ is established. This is achieved by setting a temperature difference of $20 \mathrm{~K}$ or more between the sample surfaces and the cold electrodes. At such high $S$ values, the critical saturation for ice nucleation is exceeded by far and the particles will nucleate ice and continue to grow. The particle growth is monitored by extracting small fractions of the trapped particle population from MICE at periodic residence times and directly measuring the particle mass using a time-of-flight mass spectrometer. The high temperature difference between the electrodes and the warm surfaces has the beneficial effect that sublimation from the cold electrodes is at least $10^{3}$ times less than from the warmer sample surfaces and can be neglected. Accordingly, the sublimation rate of $\mathrm{H}_{2} \mathrm{O}$ molecules from the warm sample surfaces held at $T_{\mathrm{S}}$ determines the ice particle growth rate. The ice on these surfaces constitutes the sample of interest, which for each measurement has been kept at a constant temperature between 135 and $160 \mathrm{~K}$. We use the measured particle mass growth rates to calculate the temperature-dependent sublimation rate from the sample surfaces in MICE and convert them directly into a saturation vapor pressure. Example measurements, a detailed description 
of the nanoparticle growth rate model and the data analysis are given in Appendix A.

\subsection{Relative vapor pressure measurements during temperature ramping with an ionization gauge $(T=166-190 \mathrm{~K})$}

In order to extend the saturation vapor pressure measurements to temperatures above $160 \mathrm{~K}$, we used an additional experimental setup and measured the relative vapor pressure difference of metastable crystalline ice and ice $I_{\mathrm{h}}$ between 166 and $190 \mathrm{~K}$. The setup consists of a vacuum chamber with a $5 \times 10^{-9}$ mbar residual gas pressure that encloses a temperature-controlled flat copper surface $\left(95 \mathrm{~cm}^{2}\right)$ onto which a roughly $15 \mu \mathrm{m}$ thick ice sample of interest is deposited from the gas phase at a deposition rate of about $8 \mathrm{~nm} \mathrm{~s}^{-1}$. Water vapor is provided from a water reservoir containing Nanopure ${ }^{\text {TM }}$ water that has been subjected to several freeze-pump-thaw cycles to remove dissolved gases from the liquid prior to deposition. In this setup, crystalline ice is produced using the same procedure as with the MICETRAPS setup, either via deposition of ASW at $100 \mathrm{~K}$ followed by crystallization during warm-up or by direct deposition at $150 \mathrm{~K}$. As a reference sample, hexagonal ice is produced by the condensation of liquid water on the target at about $270 \mathrm{~K}$ and subsequent freezing of the liquid water at about $260 \mathrm{~K}$. Following ice formation, the sample temperature is set to $150 \mathrm{~K}$ at which point cooling is turned off to allow for a slow warm-up $\left(\sim 0.5 \mathrm{~K} \mathrm{~min}^{-1}\right)$. The vapor pressure in the chamber is recorded as a function of the sample temperature between 166 and $190 \mathrm{~K}$ using a hot-cathode ionization gauge. A quadrupole mass spectrometer is used to confirm that no gases other than $\mathrm{H}_{2} \mathrm{O}$ bias the pressure readout. To avoid the systematic errors occurring in absolute vapor pressure measurements with a hot-cathode ionization gauge, we report only the relative vapor pressure of the lowtemperature-deposited samples with respect to the hexagonal ice sample using otherwise identical experimental procedures. For a more detailed description of this setup the reader is referred to Appendix B.

\section{Results}

Isothermal saturation vapor pressure measurements were performed with MICE-TRAPS in the temperature range between 133 and $160 \mathrm{~K}$. Non-isothermal measurements using the hot ionization gauge setup were performed with a temperature ramp of $0.5 \mathrm{~K} \mathrm{~min}^{-1}$ between 166 and $190 \mathrm{~K}$. The results are shown in Fig. 2 relative to the saturation vapor pressure of hexagonal ice $p_{\text {sat }}^{\mathrm{h}}$ taken from the parameterization of Murphy and Koop (Murphy and Koop, 2005), which is expected to be accurate to within $1 \%$ at the temperatures under investigation. At the beginning of each MICE-TRAPS experiment, water ice films were deposited on the surfaces

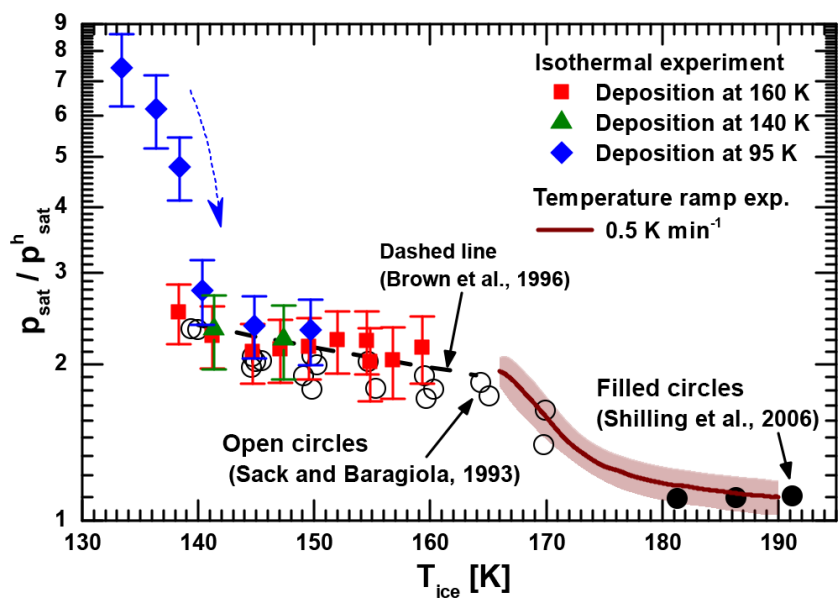

Figure 2. Measured relative saturation vapor pressure of lowtemperature-deposited ices with respect to ice $I_{\mathrm{h}}$ as a function of temperature. Green triangles and red squares denote isothermal vapor pressure measurements of ice samples deposited at 140 and $160 \mathrm{~K}$, respectively. Blue diamonds represent a series of subsequent isothermal measurements using a single ice film deposited at $95 \mathrm{~K}$ with the arrow indicating the chronology. The brown line is the combined experimental result for the non-isothermal relative vapor pressure measurements of all ices deposited below $150 \mathrm{~K}$ (including a shaded interval of uncertainty). For comparison, we show data obtained for crystalline ice from the literature (see text in figure).

in MICE either at 95, 140 or $160 \mathrm{~K}$. After ice deposition was completed, the temperature of the ice sample of interest was set to the desired temperature and isothermal measurements as described in Sect. 2.1 and Appendix A were carried out. The results are presented in Fig. 2 by the blue diamonds, green triangles and red squares. Temperature error bars are of the same size as the data points $(\Delta T=0.2$ $0.4 \mathrm{~K}$ ). The blue diamonds show the results of a series of six measurements performed using a single ASW film deposited at $95 \mathrm{~K}$ with the arrow indicating the chronology. The series started at $133.4 \mathrm{~K}$ with the freshly deposited film followed by a repeated sequence of setting the desired sample temperature and $20 \mathrm{~min}$ of thermalization followed by the measurement of particle growth at a constant temperature. For this set of measurements, we observe a sharply decreasing relative vapor pressure between 133 and $140 \mathrm{~K}$ (the first four data points), which levels off to the saturation vapor pressure obtained for the samples deposited at 140 and $160 \mathrm{~K}$ (green triangles and red squares, respectively). We interpret this behavior as the thermally activated crystallization of ASW. From our data we estimate the crystallization constant $\tau$ to be about $25 \mathrm{~min}$ at $140 \mathrm{~K}$, which is in agreement with previously reported temperature-dependent crystallization constants and times (Dowell and Rinfret, 1960; Mitchell et al., 2017; Sack and Baragiola, 1993; Smith et al., 1996; Smith et al., 2011). 
Above $140 \mathrm{~K}$, the saturation vapor pressure is found to be independent of the deposition temperature, suggesting that ice deposited between 140 and $160 \mathrm{~K}$ forms the same ice polymorph as ice crystallized from ASW. Between 135 and $160 \mathrm{~K}$ the vapor pressure of this ice polymorph is elevated by a factor between 2 and 3 with respect to hexagonal ice $I_{\mathrm{h}}$. At higher temperatures between 166 and $190 \mathrm{~K}$, the saturation vapor pressure of vapor-deposited ice was measured using the hot ionization gauge setup. As reported above for the MICE experiment, we find that ice crystallized from ASW after deposition at $100 \mathrm{~K}$ and ice deposited at $150 \mathrm{~K}$ does not show any significant difference in vapor pressure in this temperature range (see Appendix B). The average of the relative vapor pressures obtained for all runs performed with the hot ionization gauge setup is shown as a brown line in Fig. 2 with the shaded area indicating 1 standard deviation. Above $180 \mathrm{~K}$, our measurements show an only slightly elevated vapor pressure between 3 and $30 \%$ above that of hexagonal ice. At lower temperatures, however, the measured normalized vapor pressure of the crystalline ice phase increases and connects well to the vapor pressure measured with the MICETRAPS setup at $160 \mathrm{~K}$. It is noteworthy that for an ice layer thickness of several micrometers as was studied here we do not see any difference between substrate materials (gold in MICE-TRAPS and copper in the ionization gauge setup).

\section{Discussion}

\subsection{Comparison to literature data}

We reviewed and partially reanalyzed the limited amount of available literature data on the desorption rate of metastable ice below about $170 \mathrm{~K}$ in order to compare them with our measurements. These measurements typically employed a quadrupole mass spectrometer (QMS) and/or a quartz crystal microbalance to measure desorption rates. Desorption rates can be used to infer saturation vapor pressures under the well-supported assumption that the sticking coefficient for water molecules on water ice is unity at these temperatures (Batista et al., 2005; Brown et al., 1996; Gibson et al., 2011; Kong et al., 2014). Measuring water vapor desorption rates at the temperatures under investigation is a challenging task and previous experiments were influenced by contamination issues, showed a very large degree of scattering in the data or yielded unphysically low vapor pressures below that of ice $I_{\mathrm{h}}$ (Bryson et al., 1974; Fraser et al., 2001; La Spisa et al., 2001). Sack and Baragiola (1993) carefully avoided contributions of water molecules from external sources by shielding the ice sample with cold surfaces held at $12 \mathrm{~K}$ and measured the desorption rate at a constant temperature. We converted their data representing ice crystallized from ASW (Fig. 2 in Sack and Baragiola, 1993) to vapor pressures normalized to ice $I_{\mathrm{h}}$ and reproduce them as open circles in Fig. 2. Brown et al. measured temperature-dependent des- orption rates with a QMS (Brown et al., 1996). We converted their parameterized data to normalized vapor pressure values and show them as a black dashed line in Fig. 2. Both results match our measurements very well. The authors of both articles do not discuss potential causes of the measured elevated vapor pressure with respect to ice $I_{\mathrm{h}}$. However, in the case of Brown et al. (1996) a temperature error of $2 \mathrm{~K}$ is assumed, which is large enough to make their results agree with the vapor pressure of ice $I_{\mathrm{h}}$ within the limits of error. Sack and Baragiola (1993) do not discuss the temperature uncertainty of their sample and the accuracy of their measurements in detail. Comparing the results of Brown et al. (1996) and Sack and Baragiola (1993) to the results of the MICE-TRAPS experiments below $160 \mathrm{~K}$, we assume that the reported desorption rates in both publications are rather accurate and support our measurements of an elevated vapor pressure with respect to ice $I_{\mathrm{h}}$ between a factor of 2 and 3 .

Hexagonal ice is the lowest energy phase of solid water below the freezing point under typical terrestrial atmospheric conditions. The overall thermodynamic model of ice $I_{\mathrm{h}}$ is consistent and is supported by data obtained from a variety of different experiments (e.g., Feistel and Wagner, 2006, 2007; Murphy and Koop, 2005). Below about 200 K, however, water may be encountered in the metastable cubic form ice $I_{c}$ (e.g., Hobbs, 1974). Recently, studies using diffraction measurements and numerical simulations showed that samples were not composed of pure cubic ice, but rather exhibited crystalline sequences of cubic ice interlaced with sequences of hexagonal ice (e.g., Hudait et al., 2016; Kuhs et al., 2012; Lupi et al., 2017; Malkin et al., 2015; Murray et al., 2015; Shallcross and Carpenter, 1957; Thürmer and Nie, 2013). This ice polymorph has been termed stacking disordered ice $I_{\mathrm{sd}}$. It is metastable and eventually transforms to the stable ice $I_{\mathrm{h}}$. Cubic ice and hexagonal ice are both based on stacked layers of water molecules in sixfold symmetry, differing only in the stacking sequence of these layers. Hence, most physical properties of cubic and hexagonal ice are quite similar (Kuhs et al., 2012). Consequently, the vapor pressure of ice $I_{\mathrm{sd}}$ is expected to be only slightly higher compared to ice $I_{\mathrm{h}}$. In general, the higher vapor pressure $p_{\text {sat }}^{\mathrm{m}}$ of such a metastable ice polymorph compared to the vapor pressure of hexagonal ice $p_{\text {sat }}^{\mathrm{h}}$ is reflected by a Gibbs free energy difference $\Delta G_{\mathrm{m} \rightarrow \mathrm{h}}(T)$, which can be separated into an enthalpy and an entropy contribution according to

$$
\begin{aligned}
& \frac{p_{\text {sat }}^{\mathrm{m}}}{p_{\mathrm{sat}}^{\mathrm{h}}}=\exp \left(\frac{\Delta G_{\mathrm{m} \rightarrow \mathrm{h}}}{R T}\right) \text { with } \\
& \Delta G_{\mathrm{m} \rightarrow \mathrm{h}}=\Delta H_{\mathrm{m} \rightarrow \mathrm{h}}-T \Delta S_{\mathrm{m} \rightarrow \mathrm{h}} .
\end{aligned}
$$

Under the assumption that the entropy difference $\Delta S_{\mathrm{sd} \rightarrow \mathrm{h}}$ is close to zero (e.g., Tanaka, 1998; Tanaka and Okabe, 1996), $\Delta G_{\mathrm{sd} \rightarrow \mathrm{h}}$ equals $\Delta H_{\mathrm{sd} \rightarrow \mathrm{h}}$. The transformation of ice $I_{\text {sd }}$ to ice $I_{\mathrm{h}}$ at temperatures above $180 \mathrm{~K}$ has been studied extensively with differential scanning calorimetry (DSC; e.g., Handa et al., 1986; Mayer and Hallbrucker, 1987; McMillan and Los, 
1965; Sugisaki et al., 1968). These studies determined the enthalpy difference $\Delta H_{\mathrm{sd} \rightarrow \mathrm{h}}$ between the two ice phases to be in the range of 20 to $180 \mathrm{~J} \mathrm{~mol}^{-1}$, which according to Eq. (1) corresponds to a vapor pressure difference of 1 to $18 \%$ between 130 and $190 \mathrm{~K}$. This is in agreement with direct vapor pressure measurements (black dots in Fig. 2) revealing a difference of about $10 \%$ (Shilling et al., 2006) and our results between 180 and $190 \mathrm{~K}$, but is in striking contrast to our data below $170 \mathrm{~K}$. In the following we will show that the observed elevated vapor pressure below $170 \mathrm{~K}$ can be attributed to the formation of nanoscale grains formed upon the crystallization of ASW.

\subsection{The effect of nano-crystalline ice on the vapor pressure}

It is well known that the crystallization process below $166 \mathrm{~K}$ of ASW as well as the high-pressure ices II, IV, V and IX form nano-crystalline ice (Arnold et al., 1968; Backus and Bonn, 2004; Dowell and Rinfret, 1960; Jenniskens and Blake, 1996; Kondo et al., 2007; Kuhs et al., 1987; Kumai, 1968). The formation of nano-crystallites is believed to occur by nucleation of ice embryos followed by their diffusional isotropic three-dimensional growth within the remaining ASW matrix until all amorphous water is transformed to crystalline ice. At low temperatures, the interplay of ice nucleation and ice growth leads to nanoscale crystallites (e.g., Backus and Bonn, 2004; Kondo et al., 2007). A nano-crystallite exhibits a large surface energy to volume energy ratio, resulting in an increased vapor pressure above its surface. This vapor pressure increase is described by the Kelvin equation, which at the same time corresponds to the vapor pressure increase over a macroscopic surface composed of spherical nano-grains:

$\ln \left(p_{\text {sat }}^{\text {nano }} / p_{\text {sat }}^{\text {cryst }}\right)=4 \cdot v \cdot \sigma /\left(k \cdot T \cdot d_{\text {grain }}\right)$.

Equation (2) describes the vapor pressure increase over a curved surface of spherical nano-grains with a grain diameter $d_{\text {grain }}$ consisting of crystalline ice with a bulk vapor pressure $p_{\text {sat }}^{\text {cryst }}$. Here, $k$ is the Boltzmann constant, $T$ is the temperature, $v$ is the molecular volume and $\sigma$ is the ice-vapor surface tension of the crystalline ice. We assume that the crystalline nano-grains are composed of ice $I_{\mathrm{sd}}$ as supported by model studies (Lupi et al., 2017) and X-ray diffraction experiments (Morishige et al., 2009). Since the surface tension of hexagonal and cubic ice are assumed to be very similar, we used the surface tension parameterization of hexagonal ice for ice $I_{\mathrm{sd}}\left(\sigma_{\mathrm{sd}}=0.001 \cdot(141-0.15 \cdot T[K])\left[\mathrm{N} \mathrm{m}^{-1}\right]\right.$; Hale and Plummer, 1974) and assumed an uncertainty of $10 \%$. We inferred $p_{\text {sat }}^{\text {sd }}$ using Eq. (1) and assumed a free energy difference of ice $I_{\text {sd }}$ to ice $I_{\mathrm{h}}$ of 20 to $180 \mathrm{~J} \mathrm{~mol}^{-1}$ in order to calculate the grain diameters needed to explain the observed elevated vapor pressure found in this work using Eq. (2). The results for the grain diameters calculated from the MICE-TRAPS data (black squares) and from the relative vapor pressure measurements in the temperature ramp experiment (brown line, with shaded interval of confidence) are shown in Fig. 3. Below $160 \mathrm{~K}$, estimated grain size diameters are in the range between 7 and $19 \mathrm{~nm}$. According to our measurements, the crystal size does not depend on the formation temperature below $160 \mathrm{~K}$ and remains constant over a typical measurement period of $10 \mathrm{~h}$. Small grain sizes like this have previously been observed after crystallization of vapor-deposited ASW: Jenniskens and Blake (1996) observed crystal diameters of 10 to $15 \mathrm{~nm}$ between 150 and $160 \mathrm{~K}$ and Kumai (1968) reported diameters of 5 to $30 \mathrm{~nm}$ at 113 to $143 \mathrm{~K}$ using electron diffraction. Dowell and Rinfret (1960) used X-ray diffraction and observed grain sizes of about $40 \mathrm{~nm}$. The crystallization process of deuterated water from the high-vapor-pressure ices II, IV, V and IX has been examined in several studies with neutron powder diffraction: Kuhs et al. (1987) observed mean particle diameters of $16 \mathrm{~nm}$ and Arnold et al. (1968) reported mean crystal sizes of $13 \mathrm{~nm}$. The reported crystal grain diameters are indicated in Fig. 3 by the blue areas and dots. These measurements (except for Dowell and Rinfret, 1960) agree well with our grain diameter calculations. The non-isothermal relative vapor pressure measurements suggest that above $165 \mathrm{~K}$, crystal growth is effectively activated by sublimation and recondensation at the crystal surface or by local water molecule migration across grain boundaries, which is accompanied by a decrease in the $p_{\text {sat }} / p_{\text {sat }}^{\mathrm{h}}$ ratio. This conclusion is supported by a study of Hansen et al., who measured the grain sizes of deuterated ice with neutron powder diffraction and small-angle neutron scattering as a function of temperature (Hansen et al., 2008). They report mean crystal diameters between 20 and $25 \mathrm{~nm}$ after the crystallization process with crystal sizes being stable for hours up to temperatures of about $160 \mathrm{~K}$ followed by crystal growth at higher temperatures. At temperatures between 175 and $190 \mathrm{~K}$, Kuhs et al. (2012) observed crystal sizes by SEM imaging and neutron diffraction between 50 and $200 \mathrm{~nm}$. The crystal sizes increased with temperature and match the diameters calculated from our measurements in this temperature range.

Stacking disorder in ice $I_{\mathrm{sd}}$ is expected to contribute to the free energy difference $\Delta \mathrm{G}_{\mathrm{sd} \rightarrow \mathrm{h}}$ with less than $10 \mathrm{~J} \mathrm{~mol}^{-1}$ (Hondoh et al., 1983; Hudait et al., 2016). The energy contribution of stacking disorder is therefore not high enough to explain the variations in measured Gibbs free energy differences $\Delta \mathrm{G}_{\mathrm{sd} \rightarrow \mathrm{h}}$ of 20 to $180 \mathrm{~J} \mathrm{~mol}^{-1}$ of ice $I_{\mathrm{sd}}$ at temperatures above $180 \mathrm{~K}$. Defects beyond stacking faults are proposed to explain the observed energy difference of up to $180 \mathrm{~J} \mathrm{~mol}^{-1}$ (Hudait et al., 2016). However, it is unlikely that defects make up for an energy difference of the order of $1 \mathrm{~kJ} \mathrm{~mol}^{-1}$ as observed in this study below $160 \mathrm{~K}$. We therefore conclude that an increase in defects beyond stacking faults below $180 \mathrm{~K}$ is not the major process causing the observed elevated vapor pressure. In order to calculate crystal diameters, we assumed that the crystallites are 


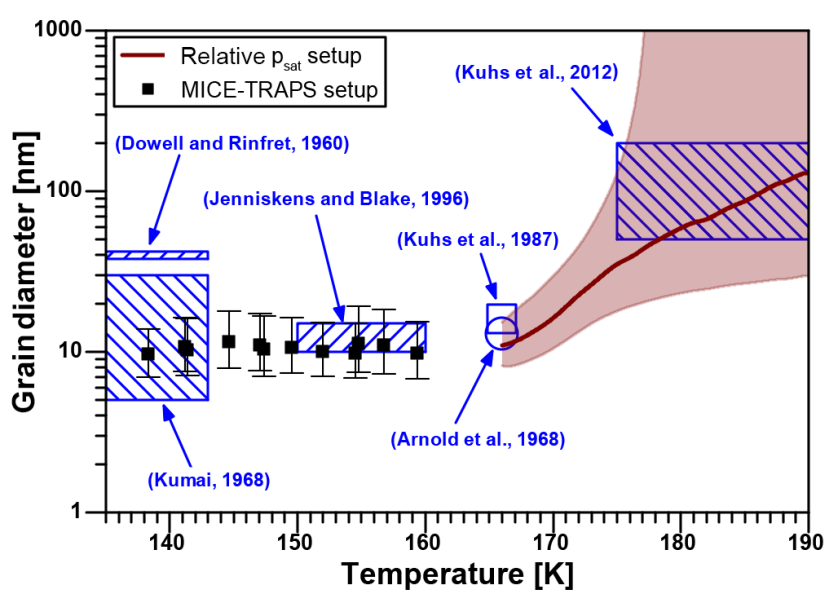

Figure 3. Calculated nano-crystallite diameters as a function of temperature in ice crystallized from ASW. The black squares (ם) represent the results of the MICE-TRAPS measurements and the brown curve with shaded confidence interval the results of the relative vapor pressure measurements. The results are compared to crystal diameters reported in the literature (blue shaded areas and dots).

composed of ice $I_{\text {sd }}$ and that this ice polymorph is described by a temperature-independent Gibbs free energy difference $\Delta G_{\mathrm{sd} \rightarrow \mathrm{h}}$ of 20 to $180 \mathrm{~J} \mathrm{~mol}^{-1}$. An increase in defects beyond stacking faults in the ice $I_{\text {sd }}$ polymorph with decreasing temperature might still cause a small increase in $\Delta G_{\mathrm{sd} \rightarrow \mathrm{h}}$, which would lead to a change in calculated crystallite sizes. However, we used the vapor pressure measurements below $160 \mathrm{~K}$ and calculated the Gibbs free energy difference of the nanocrystalline ice with respect to ice $I_{\mathrm{h}}$, which turned out to be a constant value of $\Delta G_{n \rightarrow \mathrm{h}}=(982 \pm 182) \mathrm{Jmol}^{-1}$. A significant change in $\Delta G_{\mathrm{sd} \rightarrow \mathrm{h}}$ with decreasing temperature should be directly seen in a slope of the $\Delta G_{n \rightarrow \mathrm{h}}$ values, which is not observed. Because of that and since our calculations of crystal sizes as a function of temperature are in striking agreement with previously reported values, we conclude that the increased vapor pressure below about $170 \mathrm{~K}$ is of morphological origin and can be explained solely by the wellestablished formation of nanoscale grains. The grains are stable for several hours below $160 \mathrm{~K}$ and thus nano-crystalline ice can be regarded as an independent phase at these temperatures, which is described by a constant Gibbs free energy difference of $\Delta G_{n \rightarrow \mathrm{h}}=(982 \pm 182) \mathrm{Jmol}^{-1}$ in respect to hexagonal ice.

Since deposition between 140 and $160 \mathrm{~K}$ and the crystallization of ASW deposited at 95 and $100 \mathrm{~K}$ leads to identical vapor pressures, it is very likely that ice deposition up to $160 \mathrm{~K}$ proceeds by an initial deposition of ASW followed by rapid crystallization. This conclusion is supported by the work of Chonde et al. They used deposition rates comparable to our work and observed nonporous ASW immediately after deposition at $140 \mathrm{~K}$ (Chonde et al., 2006). At temperatures above $140 \mathrm{~K}$, we cannot observe the crystallization process after deposition of ASW with the MICE-TRAPS setup since the time needed to perform the first experimental run exceeds the crystallization time at these temperatures.

It is well known that ASW might be deposited in a porous form, which depends on deposition angle, rate and temperature (Dohnalek et al., 2003; Hill et al., 2016; Kimmel et al., 2001a, b; Kouchi et al., 1994; Mayer and Pletzer, 1986; Mitterdorfer et al., 2014; Raut et al., 2007; Stevenson et al., 1999). Deposition of ASW at temperatures between 90 and $110 \mathrm{~K}$ revealed either small degrees of porosity (Brown et al., 1996; Chonde et al., 2006) or were nonporous (Kimmel et al., 2001b; Stevenson et al., 1999). Thus, reports of porosity in ASW deposited in conditions comparable to our studies are inconsistent and we cannot exclude a small degree of porosity in our ASW samples. However, due to the fact that independent of deposition temperature the same crystalline ice polymorph forms, we conclude that either all our ASW samples are nonporous or that any porosity of the ASW sample deposited at 95 and $100 \mathrm{~K}$ has no influence on the crystallized ice polymorph. The latter is supported by the observation of a strong decrease in the porosity of microporous ASW at annealing temperatures above $100 \mathrm{~K}$ with a complete absence of micropores above temperatures of $140 \mathrm{~K}$ (Hill et al., 2016; Kimmel et al., 2001b; Raut et al., 2007).

\section{Conclusions}

We present saturation vapor pressure measurements of water ices deposited from the vapor phase at temperatures below $160 \mathrm{~K}$ using two independent and complementary experimental approaches. One experiment is based on a novel technique using nanoparticles as sensitive probes for isothermal absolute sublimation rate measurements (135-160 K), and a more conventional setup uses a hot ionization gauge for relative vapor pressure measurements during a temperature ramp experiment (166-190 K).

Our vapor pressure measurements below $160 \mathrm{~K}$ show a 2 to 3 times higher saturation vapor pressure compared to ice $I_{\mathrm{h}}$. These results are consistent with previously reported measurements (Brown et al., 1996; Sack and Baragiola, 1993). The observed high vapor pressure can be quantitatively explained with the high surface energy to volume energy ratio of nanoscale crystallites (Kelvin effect). A transition in the vapor pressure data above $165 \mathrm{~K}$ is consistent with the thermally activated relaxation of nano-crystalline to stacking disordered ice of larger grain size, thereby gradually reducing the Kelvin effect. Above $180 \mathrm{~K}$, the measured saturation vapor pressure levels off at values representative for ice $I_{\mathrm{sd}}$ at these temperatures.

Because the same nano-crystalline ice polymorph forms by vapor deposition below $160 \mathrm{~K}$ and by crystallization from ASW, we conclude that even at temperatures as high as $160 \mathrm{~K}$, amorphous ice is the initial phase formed by ice deposition from the vapor prior to crystallization. This is im- 
portant for ice cloud processes that occur below $160 \mathrm{~K}$ as it implies that ice nucleation rates at these temperatures are dominated by the properties of ASW rather than those of crystalline ice. After crystallization, however, ice growth processes are described by the properties of nano-crystalline ice. The mean crystallite size of 7 to $19 \mathrm{~nm}$ in diameter determined in this work is stable for hours below $160 \mathrm{~K}$. We therefore propose considering nano-crystalline ice as an independent phase in ice cloud processes below $160 \mathrm{~K}$. For practical reasons, we provide a parameterization for the saturation vapor pressure over this ice polymorph and suggest it to be used in a temperature range in which the transformation time to microscopic crystal sizes is long compared to the processes involved. Below $160 \mathrm{~K}, p_{\text {sat }}^{\text {nano }}$ may be parameterized using a constant Gibbs free energy difference of $\Delta G_{n \rightarrow \mathrm{h}}=$ $(982 \pm 182) \mathrm{Jmol}^{-1}$ relative to the well-established parameterization for hexagonal ice (Murphy and Koop, 2005).

Our findings are of importance for cloud processes in the middle atmospheres of planets. For instance, water ice clouds are frequently observed in the middle atmosphere of Mars (Guzewich et al., 2013; Vincendon et al., 2011) with temperatures commonly falling below $160 \mathrm{~K}$ (Maltagliati et al., 2011). In the terrestrial atmosphere, noctilucent clouds form at the high-latitude summer mesopause (Rapp and Lübken, 2004) with temperatures falling to $120 \mathrm{~K}$ on average (Lübken et al., 2009) with extremes down to $100 \mathrm{~K}$ (Rapp et al., 2002). In addition, the vapor pressure of nano-crystalline ice is important for modeling $\mathrm{H}_{2} \mathrm{O}$ adsorption and desorption processes in interstellar environments and water residence times on interstellar grains (Fraser et al., 2001).

Data availability. All data are available on request from the corresponding author. 


\section{Appendix A: Nanoparticle growth model}

In general, the mass growth rate $d \mathrm{~m} / d \mathrm{t}$ of ice particles exposed to water vapor can be expressed as the difference of the water vapor deposition rate $\left(k_{\mathrm{dep}}\right)$ on the particle surface and the sublimation rate $\left(k_{\text {sub }}\right)$ from the particle surface:

$\frac{\mathrm{d} m}{\mathrm{~d} t}=\left[k_{\mathrm{dep}}-k_{\mathrm{sub}}\right] \cdot m_{\mathrm{H}_{2} \mathrm{O}}$

with $m_{\mathrm{H}_{2} \mathrm{O}}$ being the mass of one water molecule. To avoid later complications and uncertainties due to sublimation from the particle surface, MICE was operated in the experiments presented here in conditions of very high supersaturation of $S=1000$ and above where $k_{\text {dep }} \gg k_{\text {sub }}$. Under these conditions, sublimation from the particle surface can be neglected. In MICE, high supersaturation is achieved by setting a temperature difference of $20 \mathrm{~K}$ or more between the sample surfaces and the cold electrodes. This has the beneficial effect that sublimation from the electrodes is at least $10^{3}$ times less than from the sample surfaces and can be neglected, which simplifies the calculation of the deposition rate. Under the well-supported assumption of a sticking probability of unity for water molecules on water ice under the experimental conditions employed here (Batista et al., 2005; Brown et al., 1996; Gibson et al., 2011; Kong et al., 2014), Eq. (A1) can be expressed in terms of the saturation vapor pressure $p_{\text {sat, }}$ over the ice sample surfaces, yielding for the particle mass growth rate

$$
\frac{\mathrm{d} m}{\mathrm{~d} t}=A_{\mathrm{c}}(t) \frac{F_{\mathrm{s}} v_{\mathrm{th}, \mathrm{s}}}{4 k T_{\mathrm{s}}} \cdot m_{\mathrm{H}_{2} \mathrm{O} \cdot p_{\mathrm{sat}, \mathrm{s}}} .
$$

Here, $A_{\mathrm{c}}=4 \pi\left(r_{\mathrm{p}}+r_{\mathrm{H}_{2} \mathrm{O}}\right)^{2}$ is the effective particle surface area assuming spherical ice particles, $k$ is the Boltzmann constant, $v_{\text {th,s }}=\sqrt{8 k T_{\mathrm{s}} / \pi m_{\mathrm{H}_{2} \mathrm{O}}}$ is the mean thermal velocity of vapor phase molecules at temperature $T_{\mathrm{s}}$ and $F_{\mathrm{S}}=$ $0.274 \pm 0.008$ is the solid angle weighting factor of the sample surfaces as seen from the particle location, which was determined by numerical calculation based on the geometry of MICE.

Figure A1a shows the measured particle mass as a function of trapping time in MICE for three exemplary measurements with sample surface temperatures of 147.4, 149.7 (particle material: $\mathrm{Fe}_{2} \mathrm{O}_{3}$ ) and $154.8 \mathrm{~K}$ (particle material: $\mathrm{SiO}_{2}$ ). The densities of ASW and hexagonal ice are very similar at the temperatures under investigation (Brown et al., 1996; Loerting et al., 2011) so that the nature of the deposited phase does not enter in the calculation. We assume spherical particles with the above densities for the nucleus and the density of hexagonal ice for the water adsorbate to calculate the particle radius as a function of time. The results are shown in Fig. A1b. The green, blue and red curves represent numerical fits of $p_{\text {sat, } \mathrm{s}}$ in Eq. (A2). For comparison, the results of model runs assuming the vapor pressure of hexagonal ice (Murphy and Koop, 2005) are shown by the dashed lines. The vapor

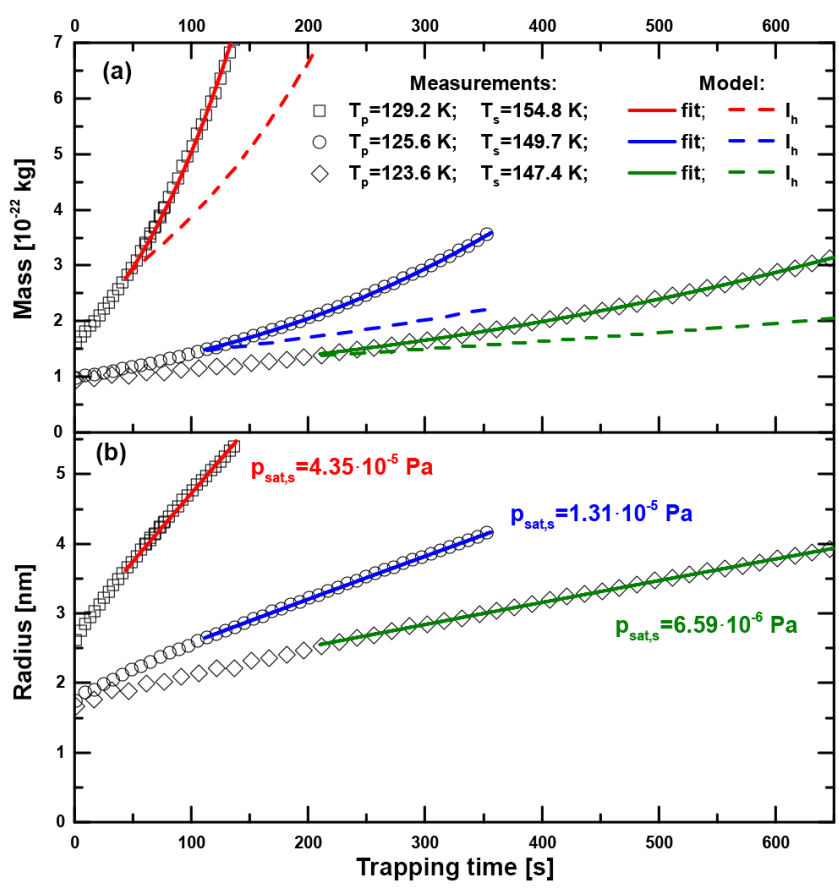

Figure A1. Particle mass (a) and radius (b) as a function of trapping time in MICE for three exemplary measurements with sample surface temperatures $T_{\mathrm{S}}$ of $147.4 \mathrm{~K}(\diamond), 149.7 \mathrm{~K}(\mathrm{\circ})$ and $154.8 \mathrm{~K}(\square)$. The particle temperatures $T_{\mathrm{p}}$ are between 123 and $130 \mathrm{~K}$, ensuring saturations above 1000 . The green, blue and red curves show the results of numerically fitting $p_{\text {sat, } \mathrm{s}}$ in Eq. (A2) to the data. The dashed colored lines show the expected growth curves when assuming hexagonal ice $I_{\mathrm{h}}$.

pressure of the investigated ice phase is according to these curves significantly higher than the one of hexagonal ice. Fit uncertainties of $p_{\text {sat,s }}$ were typically on the order of $1 \%$. The data were evaluated using Eq. (A2) only after the particles gained at least three monolayers of $\mathrm{H}_{2} \mathrm{O}$ to avoid a possible influence of the particle material on the sublimation rate. The results reported in this work are indeed independent of the type and initial radius $(1.6-2.5 \mathrm{~nm})$ of the particles under investigation. Using one fit parameter only $\left(p_{\text {sat,s }}\right)$, the implemented growth model represents the data at all temperatures very well.

In this work, only spherical nuclei and ice particles are considered. However, noctilucent clouds (NLCs) form under conditions investigated in this work and light scattering models showed better agreement to NLC data retrieved by satellite and lidar remote sensing instruments when analyzed under the assumption of aspherical ice particle shapes (Eremenko et al., 2005; Hervig et al., 2012; Kiliani et al., 2015). At the particle temperatures investigated here (below $T_{\mathrm{p}}=$ $130 \mathrm{~K}$ ) water is most likely deposited as ASW onto the ice particles, which makes aspherical particle growth unlikely. In addition, the growth model fit does not require a changing aspect ratio to achieve very good agreement with the mea- 


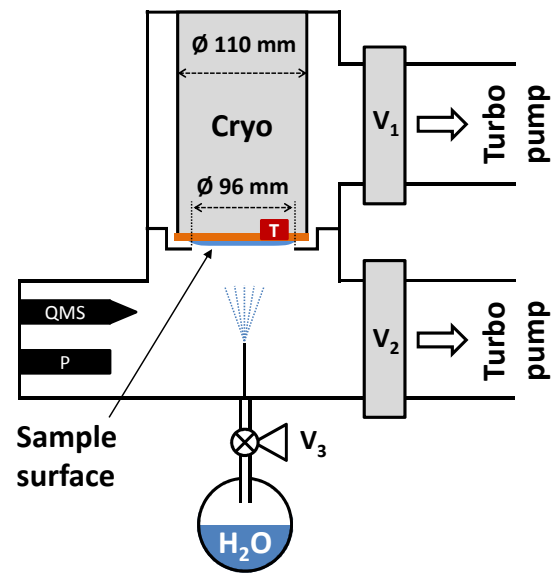

Figure B1. Experimental setup used for the relative saturation vapor pressure measurements between 166 and $190 \mathrm{~K}$. A hot-cathode ionization gauge $(P)$ is employed in a vacuum chamber, which is evacuated via valves $V_{1}$ and $V_{2}$ by two turbo molecular pumps. Simultaneous quadrupole mass spectrometer (QMS) measurements ensure that no trace gases other than $\mathrm{H}_{2} \mathrm{O}$ bias the pressure readout. See text for details.

sured data, excluding an increasing aspect ratio with particle growth. On the basis that metal-oxide nanoparticles produced in similar arrangements have been shown to be compact and spherical (Giesen et al., 2005; Janzen et al., 2002; Nadeem et al., 2012), we estimate the maximum relative uncertainty in $p_{\text {sat, } \mathrm{s}}$ due to the nonsphericity of the ice particles to $5 \%$. The main uncertainty in $p_{\text {sat, } \mathrm{s}}$ is caused by the uncertainty in $T_{\mathrm{s}}$, which is between 0.2 and $0.4 \mathrm{~K}$ depending on the applied conditions.

At $T_{\mathrm{S}}=135 \mathrm{~K}$ the time needed for a $2 \mathrm{~nm}$ radius particle to grow to a radius of about $5 \mathrm{~nm}$ is several hours. At $160 \mathrm{~K}$ the time is of the order of seconds only. These very slow and very fast growth rates set the experimental temperature limit for sublimation rate measurements with this setup.

\section{Appendix B: Relative non-isothermal vapor pressure measurements using an ionization gauge $(T=166-190 \mathrm{~K})$}

In order to extend the saturation vapor pressure measurements to temperatures above $160 \mathrm{~K}$, an additional setup to measure the relative vapor pressure difference between ice deposited below $160 \mathrm{~K}$ and hexagonal ice was built. A schematic representation of the experimental setup is depicted in Fig. B1. The setup consists of two interconnected vacuum chambers with a base residual gas pressure below $5 \times 10^{-9}$ mbar. The upper and lower chamber can be evacuated via independent valves $V_{1}$ and $V_{2}$ by two turbo molecular pumps (Leybold Turbovac 350i, 290 $\mathrm{L} \mathrm{s}^{-1}$ and Agilent Varian V 300HT, 2501s ${ }^{-1}$ ). A closed-cycle helium cryostat (Advanced Research Systems, DE110 with GMX-20B)

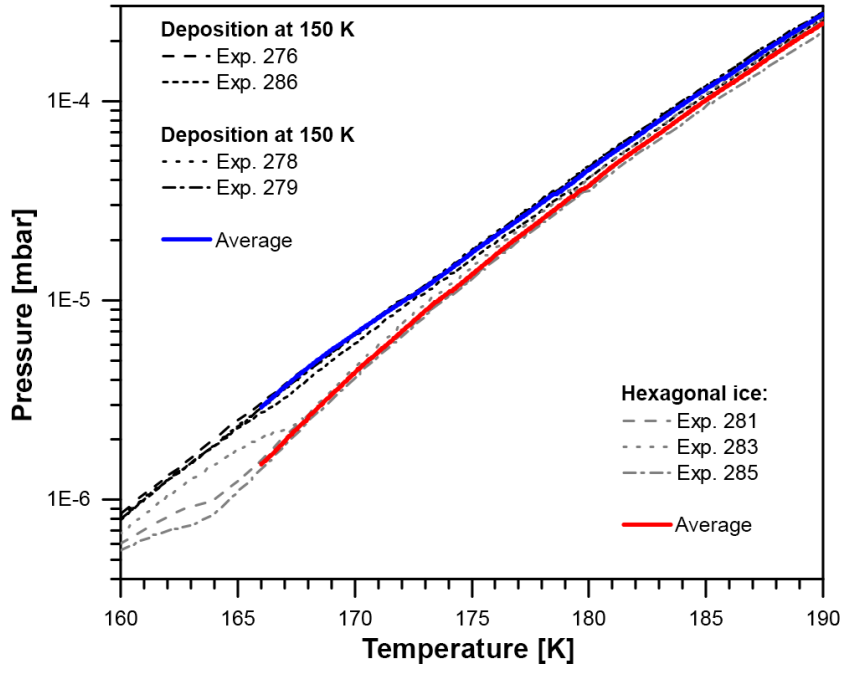

Figure B2. Vapor pressure between 160 and $190 \mathrm{~K}$ after deposition at 100 and $150 \mathrm{~K}$ (black lines, four runs) and after the crystallization of hexagonal ice from liquid water at $260 \mathrm{~K}$ (gray lines, three runs). The solid blue and red lines represent calculated mean values for deposition at 100 and $150 \mathrm{~K}$ and hexagonal ice, respectively.

is mounted in the upper chamber with the cold sample surface pointing towards the lower chamber. The sample surface is a flat and polished copper disk with a diameter of $110 \mathrm{~mm}$ and with a Pt-100 temperature sensor attached to the side of the disk. A kinked separator ring is mounted between the two chambers with an inner opening of $\varnothing=96$ and $2 \mathrm{~mm}$ of distance between the ring and cryostat. The ring serves as a barrier for water molecules from the lower to the upper chamber during the experiment. Water vapor is provided from a flask containing Nanopure ${ }^{\mathrm{TM}}$ water that has been subjected to several freeze-pump-thaw cycles to remove dissolved gases from the liquid prior to deposition. The water reservoir is connected to the vacuum chamber via the fine-dosing valve $V_{3}$ and a thin tube such that after opening the valve a deposition rate of about $8 \mathrm{~nm} \mathrm{~s}^{-1}$ on the probe is obtained.

We pursued two methods for depositing water vapor onto the sample surface.

a. Nano-crystalline ice is produced using the same procedure as with the MICE-TRAPS setup, either via the deposition of ASW at $100 \mathrm{~K}$ followed by crystallization during warm-up or by direct deposition at $150 \mathrm{~K}$ resulting in a roughly $15 \mu \mathrm{m}$ thick ice film. Both chambers were evacuated during deposition.

b. To create hexagonal ice, the fine-dosing valve was opened to the full extent with $V_{1}$ and $V_{2}$ closed and while cooling the sample surface with $2 \mathrm{~K} \mathrm{~min}^{-1}$ starting from $277 \mathrm{~K}$. At about $269 \mathrm{~K}$ the condensation of liquid water droplets could be observed by sight through a glass window mounted on the lower chamber. Sudden freezing of the water droplets was observed at about 
$260 \mathrm{~K}$ and we expect the formed ice to be hexagonal ice at this temperature. Valve $V_{3}$ to the water reservoir is closed immediately after crystallization and the probe temperature is further decreased with about $3 \mathrm{~K} \mathrm{~min}^{-1}$ down to $150 \mathrm{~K}$ with $V_{1}$ and $V_{2}$ being opened at about $210 \mathrm{~K}$. The cooling is turned off at $150 \mathrm{~K}$ to allow for a slow sample warm-up $\left(<0.5 \mathrm{~K} \mathrm{~min}^{-1}\right)$. From this point on, the measurement procedure was identical for both deposition methods.

The temperature of the sample disk was measured with a Pt100 temperature sensor and a distributed set of six Si diode sensors. It was found that during warm-up the sample surface temperature is homogeneous to within $0.2 \mathrm{~K}$ and the absolute uncertainty of the temperature measurement was estimated to be $0.5 \mathrm{~K}$. During warm-up, $V_{2}$ is closed to reduce water vapor loss by pumping. The vapor pressure of the deposited ice phase was measured as a function of the sample temperature with a hot-cathode ionization gauge $(P$; Oerlikon Leybold Ionivac ITR 90). The ITR 90 is a combined instrument comprised of a Pirani sensor for higher pressures and a BayardAlpert hot-cathode ionization sensor for lower pressures. Below $5.5 \times 10^{-3}$ mbar, which is the case for all measurements presented here, only the hot-cathode ionization sensor is active. The sensor has a characteristic curve calibrated for $\mathrm{N}_{2}$ and the pressure measured by the device can be obtained via an RS232 interface. All data in this work obtained using the ITR 90 are presented as recorded from the device without additional data processing. The data shown thus deviate from the vapor pressure above the sample surface by the $\mathrm{H}_{2} \mathrm{O}$ calibration curve of the sensor. In addition, in free molecular flow the partial pressure measured in the warm part of the chamber near the gauge $\left(T_{\mathrm{w}}\right)$ differs from the partial pressure above the cold ice sample surface $\left(T_{\mathrm{c}}\right)$ by a factor $\sqrt{T_{\mathrm{c}} / T_{\mathrm{w}}}$.

Simultaneous residual gas measurements with a quadrupole mass spectrometer (QMS; Pfeiffer PrismaPlus QMA 200) ensured that no significant amount of trace gases other than $\mathrm{H}_{2} \mathrm{O}$ bias the recorded total pressure readout. However, the data recorded by the QMS were not used to evaluate the water vapor partial pressure in this work as the QMS signal on $\mathrm{m} / \mathrm{q}$ channel 18 saturated at a temperature of about $170 \mathrm{~K}$. The resulting unprocessed recorded data of the ionization gauge are shown in Fig. B2. In total seven measurements have been performed: four times after deposition at 100 or $150 \mathrm{~K}$ (black lines) and three times after the deposition of hexagonal ice (gray lines) as described above. All four measurements of water ice deposited at 150 and $100 \mathrm{~K}$ are very close; i.e., crystalline ice deposited at $150 \mathrm{~K}$ exhibits the same vapor pressure as ice crystallized after the deposition of ASW at $100 \mathrm{~K}$. This indicates that at these temperatures independent of deposition temperature, all four samples consist of the same ice polymorph.

For hexagonal ice all curves fall onto each other above $168 \mathrm{~K}$, showing a decreasing difference towards the ices deposited at and below $150 \mathrm{~K}$. Below $168 \mathrm{~K}$, the three mea- surements of hexagonal ice show deviations, which can be explained by the following: during cooldown residual water desorbing from the inner surfaces of the vacuum chamber deposits onto the hexagonal ice film, forming a layer of the same ice that is created when depositing water directly at $150 \mathrm{~K}$. After some time of pumping and sample temperature increase, the residual water source is depleted and the layer on top of the hexagonal ice film begins to evaporate. Eventually, the overlayer will evaporate completely and expose the hexagonal ice below. The transition from one ice phase being exposed at the surface to the other can be seen in all three measurements of hexagonal ice in Fig. B2. Therefore, the analysis of the data is restricted to temperatures above $166 \mathrm{~K}$. Depending on ice thickness, all ice is evaporated somewhat above $190 \mathrm{~K}$, which limits our data to temperatures between 166 and $190 \mathrm{~K}$. Absolute vapor pressure measurements with the accuracy required to distinguish between different ice phases at such low temperatures are difficult to achieve with this setup. However, the measurements were reproducible and we can directly compare the unprocessed recorded vapor pressure of ices deposited below $160 \mathrm{~K}$ with hexagonal ice, relying for the latter on the accuracy of the well-established parameterization by Murphy and Koop (2005). In this way, we avoid many uncertainties and systematic errors occurring in absolute vapor pressure measurements. We calculated the mean and standard deviation for all runs of low-temperature vapor-deposited ice between 166 and $190 \mathrm{~K}$ (blue curve). For hexagonal ice, we use experiments 281 and 285 between 166 and $169 \mathrm{~K}$ and all three runs above $169 \mathrm{~K}$ (red curve). The recorded vapor pressures were highly reproducible and the ratio of the vapor pressures of the two ice phases could be determined with an accuracy of $10 \%$. 
Author contributions. MN and DD designed the experiments. MN carried out the MICE-TRAPS experiments. MN and DD carried out the pressure gauge experiments. MN performed the data analysis. MN prepared the paper with contributions from all coauthors. DD and TL supervised the experiments.

Competing interests. The authors declare that they have no conflict of interest.

Acknowledgements. The authors thank the German Federal Ministry of Education and Research (BMBF, grant numbers 05K13VH3 and $05 \mathrm{~K} 16 \mathrm{VHB}$ ) and the German Research Foundation (DFG, grant number LE 834/4-1) for financial support of this work. We acknowledge support by Deutsche Forschungsgemeinschaft and Open Access Publishing Fund of Karlsruhe Institute of Technology.

The article processing charges for this open-access

publication were covered by a Research

Centre of the Helmholtz Association.

Edited by: Markus Petters

Reviewed by: two anonymous referees

\section{References}

Arnold, G. P., Finch, E. D., Rabideau, S. W., and Wenzel, R. G.: Neutron-diffraction study of ice polymorphs: III. Ice ic, J. Chem. Phys., 49, 4365-4369, 1968.

Backus, E. H. G. and Bonn, M.: Theory of bulk, surface and interface phase transition kinetics in thin films, J. Chem. Phys., 121, 1038-1049, 2004.

Batista, E. R., Ayotte, P., Bilic, A., Kay, B. D., and Jonsson, H.: What determines the sticking probability of water molecules on ice?, Phys. Rev. Lett., 95, 1-4, 2005.

Brown, D. E., George, S. M., Huang, C., Wong, E. K. L., Rider, K. B., Smith, R. S., and Kay, B. D.: $\mathrm{H}_{2} \mathrm{O}$ condensation coefficient and refractive index for vapor-deposited ice from molecular beam and optical interference measurements, J. Phys. Chem., 100, 4988-4995, 1996.

Bryson, C. E., Cazcarra, V., and Levenson, L. L.: Sublimation rates and vapor-pressures of $\mathrm{H}_{2} \mathrm{O}, \mathrm{CO}_{2}, \mathrm{~N}_{2} \mathrm{O}$, and XE, J. Chem. Eng. Data, 19, 107-110, 1974.

Chonde, M., Brindza, M., and Sadtchenko, V.: Glass transition in pure and doped amorphous solid water: An ultrafast microcalorimetry study, J. Chem. Phys., 125, 1-10, 2006.

Dohnalek, Z., Kimmel, G. A., Ayotte, P., Smith, R. S., and Kay, B. D.: The deposition angle-dependent density of amorphous solid water films, J. Chem. Phys., 118, 364-372, 2003.

Dowell, L. G. and Rinfret, A. P.: Low-temperature forms of ice as studied by x-ray diffraction, Nature, 188, 1144-1148, 1960.

Duft, D., Nachbar, M., Eritt, M., and Leisner, T.: A Linear Trap for Studying the Interaction of Nanoparticles with Supersaturated Vapors, Aerosol Sci. Technol., 49, 682-690, 2015.

Eremenko, M. N., Petelina, S. V., Zasetsky, A. Y., Karlsson, B., Rinsland, C. P., Llewellyn, E. J., and Sloan, J. J.: Shape and com- position of PMC particles derived from satellite remote sensing measurements, Geophys. Res. Lett., 32, 1-4, 2005.

Feistel, R. and Wagner, W.: A new equation of state for $\mathrm{H}_{2} \mathrm{O}$ ice $\mathrm{Ih}$, J. Phys. Chem. Ref. Data, 35, 1021-1047, 2006.

Feistel, R. and Wagner, W.: Sublimation pressure and sublimation enthalpy of $\mathrm{H}_{2} \mathrm{O}$ ice $\mathrm{Ih}$ between 0 and $273.16 \mathrm{~K}$, Geochim. Cosmochim. Acta, 71, 36-45, 2007.

Fraser, H. J., Collings, M. P., McCoustra, M. R. S., and Williams, D. A.: Thermal desorption of water ice in the interstellar medium, Mon. Not. R Astron. Soc., 327, 1165-1172, 2001.

Gibson, K. D., Killelea, D. R., Yuan, H. Q., Becker, J. S., and Sibener, S. J.: Determination of the sticking coefficient and scattering dynamics of water on ice using molecular beam techniques, J. Chem. Phys., 134, 1-7, 2011.

Giesen, B., Wiggers, H., Kowalik, A., and Roth, P.: Formation of Si-nanoparticles in a microwave reactor: Comparison between experiments and modelling, J. Nanopart. Res., 7, 29-41, 2005.

Guzewich, S. D., Talaat, E. R., Toigo, A. D., Waugh, D. W., and McConnochie, T. H.: High-altitude dust layers on Mars: Observations with the Thermal Emission Spectrometer, J. Geophys. Res.-Planet., 118, 1177-1194, 2013.

Hale, B. N. and Plummer, P. L. M.: Molecular model for ice clusters in a supersaturated vapor, J. Chem. Phys., 61, 4012-4019, 1974.

Handa, Y. P., Klug, D. D., and Whalley, E.: Difference in energy between cubic and hexagonal ice, J. Chem. Phys., 84, 7009-7010, 1986.

Hansen, T. C., Koza, M. M., Lindner, P., and Kuhs, W. F.: Formation and annealing of cubic ice: II. Kinetic study, J. Phys.-Condes. Matter, 20, 1-14, 2008.

Hervig, M. E., Deaver, L. E., Bardeen, C. G., Russell, J. M., Bailey, S. M., and Gordley, L. L.: The content and composition of meteoric smoke in mesospheric ice particles from SOFIE observations, J. Atmos. Sol.-Terr. Phys., 84-85, 1-6, 2012.

Hill, C. R., Mitterdorfer, C., Youngs, T. G. A., Bowron, D. T., Fraser, H. J., and Loerting, T.: Neutron Scattering Analysis of Water's Glass Transition and Micropore Collapse in Amorphous Solid Water, Phys. Rev. Lett., 116, 1-5, 2016.

Hobbs, P. V.: Ice Physics, Oxford University Press, Oxford, 837 pp., 1974.

Hondoh, T., Itoh, T., Amakai, S., Goto, K., and Higashi, A.: Formation and annihilation of stacking faults in pure ice, J. Phys. Chem., 87, 4040-4044, 1983.

Hudait, A., Qiu, S. W., Lupi, L., and Molinero, V.: Free energy contributions and structural characterization of stacking disordered ices, Phys. Chem. Chem. Phys., 18, 9544-9553, 2016.

Janzen, C., Kleinwechter, H., Knipping, J., Wiggers, H., and Roth, P.: Size analysis in low-pressure nanoparticle reactors: comparison of particle mass spectrometry with in situ probing transmission electron microscopy, J. Aerosol. Sci., 33, 833-841, 2002.

Jenniskens, P. and Blake, D. F.: Crystallization of amorphous water ice in the solar system, Astrophys. J., 473, 1104-1114, 1996.

Johari, G. P. and Andersson, O.: Effects of stacking disorder on thermal conductivity of cubic ice, J. Chem. Phys., 143, 1-11, 2015.

Kiliani, J., Baumgarten, G., Lübken, F.-J., and Berger, U.: Impact of particle shape on the morphology of noctilucent clouds, Atmos. Chem. Phys., 15, 12897-12907, https://doi.org/10.5194/acp-1512897-2015, 2015.

Kimmel, G. A., Dohnalek, Z., Stevenson, K. P., Smith, R. S., and Kay, B. D.: Control of amorphous solid water morphology using 
molecular beams: II. Ballistic deposition simulations, J. Chem. Phys., 114, 5295-5303, 2001a.

Kimmel, G. A., Stevenson, K. P., Dohnalek, Z., Smith, R. S., and Kay, B. D.: Control of amorphous solid water morphology using molecular beams: I. Experimental results, J. Chem. Phys., 114, 5284-5294, 2001b.

Kondo, T., Kato, H. S., Bonn, M., and Kawai, M.: Morphological change during crystallization of thin amorphous solid water films on Ru(0001), J. Chem. Phys., 126, 1-5, 2007.

Kong, X., Papagiannakopoulos, P., Thomson, E. S., Marković, N., and Pettersson, J. B. C.: Water Accommodation and Desorption Kinetics on Ice, J. Phys. Chem. A, 118, 3973-3979, 2014.

Kouchi, A., Yamamoto, T., Kozasa, T., Kuroda, T., and Greenberg, J. M.: Conditions for condensation and preservation of amorphous ice and crystallinity of astrophysical ices, Astron. Astrophys., 290, 1009-1018, 1994.

Kuhs, W. F., Bliss, D. V., and Finney, J. L.: High-resolution neutron powder diffraction study of ice-Ic, J. Phys., 48, 631-636, 1987.

Kuhs, W. F., Sippel, C., Falenty, A., and Hansen, T. C.: Extent and relevance of stacking disorder in "ice I-c", P. Natl. Acad. Sci. USA, 109, 21259-21264, 2012.

Kumai, M.: Hexagonal and Cubic Ice at Low Temperatures, J. Glaciol., 7, 95-108, 1968.

La Spisa, S., Waldheim, M., Lintemoot, J., Thomas, T., Naff, J., and Robinson, M.: Infrared and vapor flux studies of vapor-deposited amorphous and crystalline water ice films between 90 and 145 K, J. Geophys. Res.-Planet., 106, 33351-33361, 2001.

Loerting, T., Bauer, M., Kohl, I., Watschinger, K., Winkel, K., and Mayer, E.: Cryoflotation: Densities of Amorphous and Crystalline Ices, J. Phys. Chem. B, 115, 14167-14175, 2011.

Lübken, F. J., Lautenbach, J., Höffner, J., Rapp, M., and Zecha, M.: First continuous temperature measurements within polar mesosphere summer echoes, J. Atmos. Sol.-Terr. Phys., 71, 453-463, 2009.

Lupi, L., Hudait, A., Peters, B., Grünwald, M., Gotchy Mullen, R., Nguyen, A. H., and Molinero, V.: Role of stacking disorder in ice nucleation, Nature, 551, 218-222, 2017.

Malkin, T. L., Murray, B. J., Salzmann, C. G., Molinero, V., Pickering, S. J., and Whale, T. F.: Stacking disorder in ice I, Phys. Chem. Chem. Phys., 17, 60-76, 2015.

Maltagliati, L., Montmessin, F., Fedorova, A., Korablev, O., Forget, F., and Bertaux, J. L.: Evidence of Water Vapor in Excess of Saturation in the Atmosphere of Mars, Science, 333, 1868-1871, 2011.

Mayer, E. and Hallbrucker, A.: Cubic ice from liquid water, Nature, 325, 601-602, 1987.

Mayer, E. and Pletzer, R.: Astrophysical implications of amorphous ice - a microporous solid, Nature, 319, 298-301, 1986.

McMillan, J. A. and Los, S. C.: Vitreous ice - irreversible transformations during warm-up, Nature, 206, 806-807, 1965.

Meinen, J., Khasminskaya, S., Rühl, E., Baumann, W., and Leisner, T.: The TRAPS Apparatus: Enhancing Target Density of Nanoparticle Beams in Vacuum for X-ray and Optical Spectroscopy, Aerosol Sci. Technol., 44, 316-328, 2010.

Mitchell, E. H., Raut, U., Teolis, B. D., and Baragiola, R. A.: Porosity effects on crystallization kinetics of amorphous solid water: Implications for cold icy objects in the outer solar system, Icarus, 285, 291-299, 2017.
Mitterdorfer, C., Bauer, M., Youngs, T. G. A., Bowron, D. T., Hill, C. R., Fraser, H. J., Finney, J. L., and Loerting, T.: Small-angle neutron scattering study of micropore collapse in amorphous solid water, Phys. Chem. Chem. Phys., 16, 16013-16020, 2014.

Morishige, K., Yasunaga, H., and Uematsu, H.: Stability of Cubic Ice in Mesopores, J. Phys. Chem. C, 113, 3056-3061, 2009.

Murphy, D. M. and Koop, T.: Review of the vapour pressures of ice and supercooled water for atmospheric applications, Q. J. Roy. Meteor. Soc., 131, 1539-1565, 2005.

Murray, B. J., Malkin, T. L., and Salzmann, C. G.: The crystal structure of ice under mesospheric conditions, J. Atmos. Sol.-Terr. Phys., 127, 78-82, 2015.

Nachbar, M., Duft, D., Kiselev, A., and Leisner, T.: Composition, Mixing State and Water Affinity of Meteoric Smoke Analogue Nanoparticles Produced in a Non-Thermal Microwave Plasma Source, in: Zeitschrift für Physikalische Chemie, 1-14, 2018.

Nachbar, M., Duft, D., Mangan, T. P., Martin, J. C. G., Plane, J. M. C., and Leisner, T.: Laboratory measurements of heterogeneous $\mathrm{CO}_{2}$ ice nucleation on nanoparticles under conditions relevant to the Martian mesosphere, J. Geophys. Res.-Planet., 121, 753-769, 2016.

Nadeem, K., Krenn, H., Traussnig, T., Wurschum, R., Szabo, D. V., and Letofsky-Papst, I.: Spin-glass freezing of maghemite nanoparticles prepared by microwave plasma synthesis, J. Appl. Phys., 111, 1-6, 2012.

Rapp, M. and Lübken, F.-J.: Polar mesosphere summer echoes (PMSE): Review of observations and current understanding, Atmos. Chem. Phys., 4, 2601-2633, https://doi.org/10.5194/acp-42601-2004, 2004.

Rapp, M., Lübken, F. J., Müllemann, A., Thomas, G. E., and Jensen, E. J.: Small-scale temperature variations in the vicinity of NLC: Experimental and model results, J. Geophys. Res.-Atmos., 107, 4392, https://doi.org/10.1029/2001JD001241, 2002.

Raut, U., Fama, M., Teolis, B. D., and Baragiola, R. A.: Characterization of porosity in vapor-deposited amorphous solid water from methane adsorption, J. Chem. Phys., 127, 1-6, 2007.

Sack, N. J. and Baragiola, R. A.: Sublimation of vapor-deposited water ice below $170 \mathrm{~K}$, and its dependence on growth-conditions, Phys. Rev. B, 48, 9973-9978, 1993.

Shallcross, F. V. and Carpenter, G. B.: X-Ray Diffraction Study of the Cubic Phase of Ice, J. Chem. Phys., 26, 782-784, 1957.

Shilling, J. E., Tolbert, M. A., Toon, O. B., Jensen, E. J., Murray, B. J., and Bertram, A. K.: Measurements of the vapor pressure of cubic ice and their implications for atmospheric ice clouds, Geophys. Res. Lett., 33, L17801, https://doi.org/10.1029/2006GL026671, 2006.

Smith, R. S., Huang, C., Wong, E. K. L., and Kay, B. D.: Desorption and crystallization kinetics in nanoscale thin films of amorphous water ice, Surf. Sci., 367, L13-L18, 1996.

Smith, R. S., Matthiesen, J., Knox, J., and Kay, B. D.: Crystallization Kinetics and Excess Free Energy of $\mathrm{H}_{2} \mathrm{O}$ and $\mathrm{D}_{2} \mathrm{O}$ Nanoscale Films of Amorphous Solid Water, J. Phys. Chem. A, 115, 5908-5917, 2011.

Speedy, R. J., Debenedetti, P. G., Smith, R. S., Huang, C., and Kay, B. D.: The evaporation rate, free energy, and entropy of amorphous water at 150 K, J. Chem. Phys., 105, 240-244, 1996.

Stevenson, K. P., Kimmel, G. A., Dohnalek, Z., Smith, R. S., and Kay, B. D.: Controlling the morphology of amorphous solid water, Science, 283, 1505-1507, 1999. 
Sugisaki, M., Suga, H., and Seki, S.: Calorimetric study of glassy state .4. Heat capacities of glassy water and cubic ice, Bull. Chem. Soc. Jpn., 41, 2591-2599, 1968.

Tanaka, H.: Thermodynamic stability and negative thermal expansion of hexagonal and cubic ices, J. Chem. Phys., 108, 48874893, 1998.

Tanaka, H. and Okabe, I.: Thermodynamic stability of hexagonal and cubic ices, Chem. Phys. Lett., 259, 593-598, 1996.
Thürmer, K. and Nie, S.: Formation of hexagonal and cubic ice during low-temperature growth, P. Natl. Acad. Sci. USA, 110, 11757-11762, 2013.

Vincendon, M., Pilorget, C., Gondet, B., Murchie, S., and Bibring, J. P.: New near-IR observations of mesospheric $\mathrm{CO}_{2}$ and $\mathrm{H}_{2} \mathrm{O}$ clouds on Mars, J. Geophys. Res.-Planets, 116, 1-18, 2011. 